International Journal of Quantum Information

(C) World Scientific Publishing Company

\title{
LANDAU-ZENER TRANSITIONS IN THE PRESENCE OF SPIN ENVIRONMENT
}

\author{
ANDY T. S. WAN*, M. H. S. AMIN, and SHANNON X. WANG ${ }^{\dagger}$ \\ D-Wave Systems Inc., 100-4401 Still Creek Drive, \\ Burnaby, B.C., V5C 6G9, Canada
}

\begin{abstract}
We study the effect of an environment consisting of noninteracting two level systems on Landau-Zener transitions with an interest on the performance of an adiabatic quantum computer. We show that if the environment is initially at zero temperature, it does not affect the transition probability. An excited environment, however, will always increase the probability of making a transition out of the ground state. For the case of equal intermediate gaps, we find an analytical upper bound for the transition probability in the limit of large number of environmental spins. We show that such an environment will only suppress the probability of success for adiabatic quantum computation by at most a factor close to $1 / 2$.
\end{abstract}

Keywords: Adiabatic quantum computation; spin environment; decoherence.

\section{Introduction}

Theories of open quantum systems have gained renewed attention in recent years, thanks to their important role in quantum computation. Such theories usually study quantum evolution of a system in contact with an environment, which in general can be made of bosons 1 , fermions ${ }^{2}$, or localized spins 3 . At the end of the evolution, the properties of the system are extracted by averaging over the environmental degrees of freedom.

Significant progress has been made in understanding the effect of environment on quantum coherence and quantum computation 4516 . There is evidence that certain quantum computational models are less sensitive to loss of phase coherence than others. One of those, is adiabatic quantum computation (AQC) $7|8| 9$. In AQC, the system starts in a known ground state of a Hamiltonian $H_{i}$. The Hamiltonian then slowly evolves to a final Hamiltonian $H_{f}$, whose ground state encodes the solution to the problem. In the absence of an environment, if the evolution is slow enough, the system will end up in the ground state of $H_{f}$ with probability close to one. The total time dependent system Hamiltonian is usually written as a linear interpolation

$$
H_{S}(t)=[1-s(t)] H_{i}+s(t) H_{f},
$$

* Currently at UBC mathematics department.

${ }^{\dagger}$ Currently at MIT physics department. 
where $s(t) \in[0,1]$ is a monotonic function of time $t$.

In the absence of an environment, the main source of error for AQC is nonadiabatic transition out of the ground state. In the small gap regime, it is closely related to the Landau-Zener (LZ) transitions 10 , which occur when two energy levels pass an avoided crossing (anticrossing). In the original LZ problem, a 2-level system evolves via a Hamiltonian $H=-\left(\Delta \tau^{x}+\nu t \tau^{z}\right) / 2$, where $\tau^{x, z}$ are the Pauli matrices. The spectrum of this system has an anticrossing at $t=0$ with gap $\Delta$. If at $t \rightarrow-\infty$ the system is in its ground state, the probability of finding it in its excited state at time $t \rightarrow \infty$ is exactly given by 10

$$
P_{\mathrm{LZ}}=e^{-\pi \Delta^{2} / 2 \nu}
$$

In AQC, the system has many energy levels, and the running time is finite; therefore the above problem does not directly apply. However, when the gap $g_{m}$ between the first two levels is much smaller than other relevant energy scales, the transition probability is given by (2) to a very good approximation. In such a case, $\nu \propto 1 / t_{f}$, where $t_{f}$ is the running time. In order to have small excitation probability, one needs a long $t_{f}\left(\propto 1 / g_{m}^{2}\right)$. Therefore, problems with small gap are the hardest to solve using AQC.

Recently, there has been compelling evidence that localized two level systems play an important role in removing phase coherence in solid state quantum systems $5 / 11 \mid 12[13$. Such 2-level systems can be nuclear spins 6 , magnetic impurities, or other 2-level fluctuators 13|14 The effect of a bosonic environment on LZ transition has been studied extensively $15|16| 17$, and some studies have been done for a spin environment $\frac{18}{}$. In this article, we study a spin environment focusing only on small gap problems, relevant for AQC.

\section{Hamiltonian}

As usual we write the total Hamiltonian as $H=H_{S}+H_{B}+H_{\text {int }}$, which includes system, bath, and interaction Hamiltonians respectively. If the minimum gap $g_{m}$ is much smaller than the separation of the two crossing levels from the other energy levels, then the slow evolution of the system close to the anticrossing will be restricted only to those two levels. Using a new coordinate $\epsilon=2 E\left(s-s^{*}\right)$, where $E$ is an energy scale characterizing the anticrossing and $s^{*}$ is its position, one can write a two-state Hamiltonian:

$$
H_{S}=-\left(\epsilon \tau_{z}+g_{m} \tau_{x}\right) / 2
$$

and the gap between the first two states is well approximated by

$$
g=\sqrt{\epsilon^{2}+g_{m}^{2}} .
$$

with $\tau_{x, z}$ being the Pauli matrices in the two-state subspace of the central system. We only focus on linear time sweep for which $\epsilon=\nu t$ with $\nu=2 E / t_{f}$. In this case, the two-state problem becomes a LZ problem. 
We incorporate the environment via the Hamiltonians

$$
H_{B}=-\frac{1}{2} \sum_{j=1}^{m} \boldsymbol{B}_{j} \cdot \boldsymbol{\sigma}_{j}, \quad H_{\mathrm{int}}=-\frac{1}{2} \sum_{j=1}^{m} J_{j} \tau^{z} \sigma_{j}^{z} .
$$

Here, $\sigma^{x, z}$ are Pauli matrices for the environmental spins, $\boldsymbol{B}_{j}=\left[B_{j}^{x}, B_{j}^{y}, B_{j}^{z}\right]$ is a local field applied to the $j$-th environmental spin, $J_{j}$ is the coupling coefficient between $j$-th spin and the system, and $m$ is the number of environmental spins. Notice that we only take into account longitudinal coupling between the effective twostate system $\left(\tau^{z}\right)$ and the environmental spins $\left(\sigma_{j}^{z}\right)$. As is shown in the appendix, in the case of adiabatic Grover search problem 20 , a general coupling between individual qubits within the system and the environmental spins leads to only a longitudinal coupling between the effective two-state system at the anticrossing and the environmental spins in the limit of large number of qubits. The same behavior is however expected for more general Hamiltonians because transverse coupling to the environment will cause large relaxation to the ground state, enabling us to solve the problem efficiently by relaying only on classical relaxation (i.e., classical annealing) which is not expected.

In general, the effect of the environment is to split (broaden) each energy level into $M=2^{m}$ levels, thereby splitting the original anticrossing into $M^{2}$ avoided crossings, as shown in Fig. 1. Since we are interested in small $g_{m}$, we use a perturbative approach: $H=H_{0}+H^{\prime}$, with the perturbation Hamiltonian $H^{\prime}=-g_{m} \tau^{x} / 2$ and the unperturbed Hamiltonian $H_{0}$ which constitutes all the terms in $H$ except $H^{\prime}$. To diagonalize $H_{0}$, we introduce a unitary transformation $\mathrm{U}$ by

$$
\begin{aligned}
U= & \prod_{j=1}^{m} \frac{1}{2}\left[a_{j}+i b_{j}\left(B_{j}^{x} \sigma_{j}^{y}-B_{j}^{y} \sigma_{j}^{x}\right) / \sqrt{\left(B_{j}^{x}\right)^{2}+\left(B_{j}^{y}\right)^{2}}\right] \\
& a_{j}=\left(\cos \theta_{j}^{+}+\cos \theta_{j}^{-}\right) \mathbb{I}+\left(\cos \theta_{j}^{+}-\cos \theta_{j}^{-}\right) \tau^{z}, \\
& b_{j}=\left(\sin \theta_{j}^{+}-\sin \theta_{j}^{-}\right) \mathbb{I}+\left(\sin \theta_{j}^{+}+\sin \theta_{j}^{-}\right) \tau^{z}, \\
& \theta_{j}^{ \pm}=\arccos \sqrt{\left(\Omega_{j}^{ \pm} \pm B_{j}^{z}+J_{j}\right) / 2 \Omega_{j}^{ \pm}} \\
& \Omega_{j}^{ \pm}=\sqrt{\left(B_{j}^{x}\right)^{2}+\left(B_{j}^{y}\right)^{2}+\left(B_{j}^{z} \pm J_{j}\right)^{2}} .
\end{aligned}
$$

After the transformation, we find

$$
\begin{aligned}
U H_{0} U^{\dagger} & =\sum_{z, s_{1}, \ldots, s_{m}} E_{z s_{1} \ldots s_{m}}\left|z s_{1} \ldots s_{m}\right\rangle\left\langle z s_{1} \ldots s_{m}\right| \\
U H^{\prime} U^{\dagger} & =-\frac{1}{2} g_{m} \tau^{x} \prod_{j=1}^{m}\left[\cos \theta_{j}-i \tau^{z} \sin \theta_{j} \frac{B_{j}^{x} \sigma_{j}^{y}-B_{j}^{y} \sigma_{j}^{x}}{\sqrt{\left(B_{j}^{x}\right)^{2}+\left(B_{j}^{y}\right)^{2}}}\right] \\
E_{z s_{1} \ldots s_{m}} & =-\frac{1}{2}\left\{\epsilon(-1)^{z}+\frac{1}{2} \sum_{j=1}^{m}(-1)^{s_{j}}\left[\left(\Omega_{j}^{+}-\Omega_{j}^{-}\right)+(-1)^{z}\left(\Omega_{j}^{+}+\Omega_{j}^{-}\right)\right]\right\},
\end{aligned}
$$


where $z$ and $s_{j}$ are eigenvalues of $\left(\mathbb{I}-\tau^{z}\right) / 2$ and $\left(\mathbb{I}-\sigma_{j}^{z}\right) / 2$ respectively, and $\theta_{j} \equiv$ $\theta_{j}^{+}+\theta_{j}^{-}$.

Let $|z, k\rangle$ denote the state of the system plus the environment when the environment is in its $k$-th energy level. We use degenerate perturbation theory to find the gap $g_{k k^{\prime}}$ at the point where $|0, k\rangle$ and $\left|1, k^{\prime}\right\rangle$ cross (see Fig. 1). Assuming that $H_{0}$ has no other degeneracies except at the crossings, we find

$$
g_{k k^{\prime}}=g_{m} \prod_{j=1}^{m}\left|\cos \theta_{j} \delta_{s_{j} s_{j}^{\prime}}+\sin \theta_{j}\left(1-\delta_{s_{j} s_{j}^{\prime}}\right)\right|,
$$

where $\delta_{s_{j} s_{j}^{\prime}}$ is the Kronecker delta function. Now for any $k$, one can easily show that

$$
\begin{aligned}
\sum_{k^{\prime}=1}^{M} g_{k k^{\prime}}^{2} & =g_{m}^{2} \prod_{j=1}^{m} \sum_{s_{j}^{\prime}}\left[\cos ^{2} \theta_{j} \delta_{s_{j} s_{j}^{\prime}}+\sin ^{2} \theta_{j}\left(1-\delta_{s_{j} s_{j}^{\prime}}\right)\right] \\
& =g_{m}^{2} .
\end{aligned}
$$

This is a very important sum rule which will be used in our analysis repeatedly.

\section{Environment at zero temperature}

While the intermediate gaps (9) are proportional to $g_{m}$, their separation is independent of $g_{m}$. Therefore, in the limit $g_{m} \rightarrow 0$, there is always a regime in which the intermediate gaps can be considered separated enough to justify using LZ formula, $p_{k k^{\prime}}=e^{-\pi g_{k k^{\prime}}^{2} / 2 \nu}$, for the probability of transition at the $k k^{\prime}$-th anticrossing. One

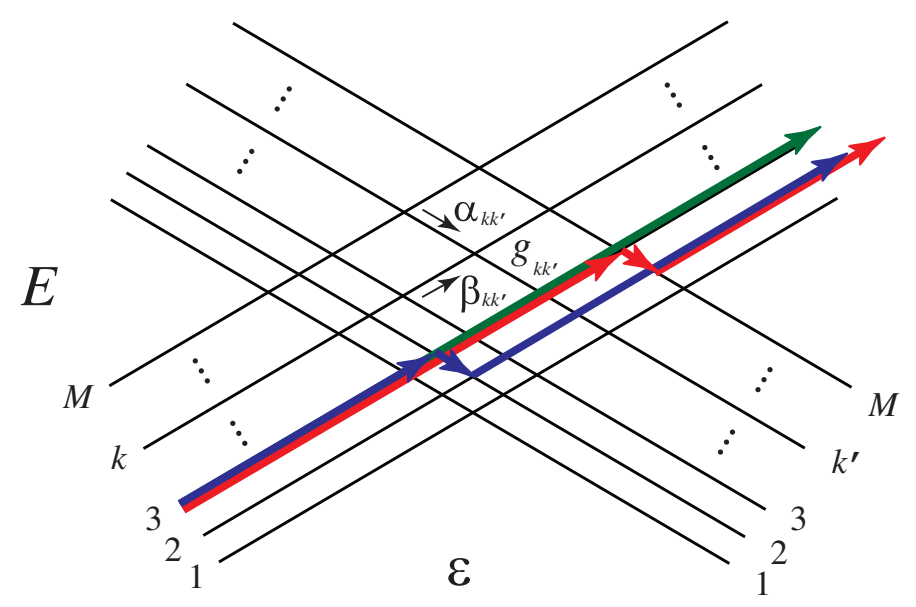

Fig. 1. Energy spectrum of the system plus the environment. The arrows show paths corresponding to excitation of the system. The green line shows a path in the fast passage limit. The red and blue lines show two interfering paths. 
immediately finds

$$
\prod_{k^{\prime}=1}^{M} p_{k k^{\prime}}=\exp \left\{-\sum_{k^{\prime}} \pi g_{k k^{\prime}}^{2} / 2 \nu\right\}=e^{-\pi g_{m}^{2} / 2 \nu}=P_{\mathrm{LZ}}
$$

where $P_{\mathrm{LZ}}$ is given by (2). Equation (11) has an important consequence when the environment is initially in its ground state $(k=1)$. The probability of ending up in the final excited state is the probability that all intermediate LZ excitations occur (straight path in Fig. 1): $P_{e f}=\prod_{k=1}^{M} p_{1 k}=P_{\mathrm{LZ}}$. Hence, a zero temperature spin environment does not affect $L Z$ transition probability. Similar results have also been reported for an environment made of uncoupled harmonic oscillators $15 \mid 17$.

\section{Environment at finite temperature}

We now discuss the effect of an excited (equilibrium or nonequilibrium) environment. Let us assume that the system plus the environment starts from its $i$-th level. A straight path (e.g., green path in Fig. 1) still yields $P_{\mathrm{LZ}}$ because of (11). But now there are many other paths to excite the system, which in general may interfere with each other (e.g., blue and red paths in Fig. 11). Those paths, however, can only add to the probability. Such property also exists if the environment is initially in a mixed state. Therefore, an initially excited (incoherent) environment will always increase the LZ transition probability. An important question now is to what extent the final ground state probability $P_{g f}$ will be suppressed as $m$ grows. If $P_{g f} \rightarrow 0$ as $m \rightarrow \infty$, then AQC will be impossible in the presence of such an environment. Fortunately, as we shall show below, this is not the case.

Phase coherence plays a role when different paths that start form a common level rejoin in another level and therefore interfere with each other. The phase that the wave function picks along each path is the time integral of the eigenenergy along that path. For very fast evolutions, all the paths come with the same phase (i.e., $\sim 0$ ). At slower evolutions, different paths will have different phases. Because of natural randomness in the parameters, the relative phases of these paths will be randomized and therefore will eliminate the effect of coherence. Thus, phase coherence is expected to have negligible effect at long times and large number of paths (i.e., large $m$ ). This is justified by our numerical simulations already at $m=4$ (see Fig. 3).

In practice, the environmental spins also are subject to thermal fluctuations, which affect the system via fluctuating local fields $\mathbf{B}_{j}$. This effect can be modeled by coupling the spins to an additional (e.g. bosonic) environment which can cause additional dephasing between the energy levels making our incoherent approach even more relevant. Such an environment does not change LZ probabilities $p_{i j}$ in the fast passage limit $\left(p_{i j} \approx 1\right), \frac{15}{15}$ which is always the case when $m \rightarrow \infty$. Here, we also neglect any relaxation effect as it can only reduce the excitation probability, i.e., enhance success probability in AQC. Hence, our calculation provides a lower bound for the AQC success probability. 
Let $\alpha_{i j}$ and $\beta_{i j}$ be the probabilities for the upper and lower energy states just before traversing the $i j$-th anticrossing, respectively (see Fig. 1). We can write

$$
\begin{aligned}
\alpha_{(i-1) j} & =p_{i j} \alpha_{i j}+\left(1-p_{i j}\right) \beta_{i j}, \\
\beta_{i(j+1)} & =p_{i j} \beta_{i j}+\left(1-p_{i j}\right) \alpha_{i j},
\end{aligned}
$$

with boundary conditions: $\alpha_{M j}=P_{e i}^{(j)}, \alpha_{0 j}=P_{g f}^{(j)}, \beta_{i 1}=P_{g i}^{(i)}$, and $\beta_{i(M+1)}=P_{e f}^{(i)}$. Here $P_{g i}^{(j)}$ and $P_{e i}^{(j)}\left(P_{g f}^{(j)}\right.$ and $\left.P_{e f}^{(j)}\right)$ are the initial (final) probabilities of finding the total system in $|0, j\rangle$ and $|1, j\rangle$, respectively. This has been studied by switching to continuous variables by Ref. 18. Here we instead solve the discrete relation while keeping the constraint (11). From (12) we get

$$
\begin{aligned}
& \boldsymbol{\alpha}_{i-1}=P_{g i}^{(i)} \boldsymbol{\mu}^{(i)}+\boldsymbol{L}^{(i)} \boldsymbol{\alpha}_{i}, \\
& \boldsymbol{\beta}_{j+1}=P_{e i}^{(j)} \boldsymbol{\eta}^{(j)}+\boldsymbol{U}^{(j)} \boldsymbol{\beta}_{j},
\end{aligned}
$$

with $\quad$ vectors $\quad \boldsymbol{\alpha}_{i}=\left[\alpha_{i 1}, \ldots, \alpha_{i M}\right]^{T}, \quad \boldsymbol{\beta}_{j}=\left[\beta_{1 j}, \ldots, \beta_{M j}\right]^{T}, \quad \mu_{j}^{(i)}=\left(1-p_{i j}\right) \prod_{l=1}^{j-1} p_{i l}$, $\eta_{i}^{(j)}=\left(1-p_{i j}\right) \prod_{l=i+1}^{M} p_{l j}$, and

$$
\begin{array}{r}
L_{j k}^{(i)}= \begin{cases}p_{i j} & k=j \\
\left(1-p_{i k}\right)\left(1-p_{i j}\right) \prod_{l=k+1}^{j-1} p_{i l} & j>k \\
0 & k>j\end{cases} \\
U_{i k}^{(j)}= \begin{cases}p_{i j} & i=k \\
\left(1-p_{k j}\right)\left(1-p_{i j}\right) \prod_{l=i+1}^{k-1} p_{l j} & k>i \\
0 & i>k\end{cases}
\end{array}
$$

We define the vectors $\boldsymbol{P}_{\boldsymbol{g} i}$ and $\boldsymbol{P}_{\boldsymbol{e} \boldsymbol{i}}\left(\boldsymbol{P}_{\boldsymbol{g} \boldsymbol{f}}\right.$ and $\left.\boldsymbol{P}_{\boldsymbol{e} \boldsymbol{f}}\right)$ to represent the initial (final) ground and excited state distribution, respectively. Here, we only consider the case where the central system is initialized in its ground state, i.e., $\boldsymbol{P}_{\boldsymbol{e} i}=\mathbf{0}$, for which we have

$$
\boldsymbol{P}_{\boldsymbol{e f}}=\boldsymbol{\Lambda} \boldsymbol{P}_{\boldsymbol{g} \boldsymbol{i}}, \quad \text { with } \boldsymbol{\Lambda}=\boldsymbol{U}^{(M)} \boldsymbol{U}^{(M-1)} \ldots \boldsymbol{U}^{(1)} .
$$

The final excited state probability $P_{e f}$ is found by summing over all elements of $\boldsymbol{P}_{\boldsymbol{e f}}$, which is equivalent to tracing over the environmental degrees of freedom.

Naturally, it is very difficult to find an analytical solution for the general case. Here, we consider a special case where all the gaps have the same size, $g_{i j}=g_{m} / \sqrt{M}$, hence $p_{i j}=p=P_{\mathrm{LZ}}^{1 / M}$. Such a situation occurs when $\left|J_{j}\right|=\left|\boldsymbol{B}_{j}\right|$. We find $\boldsymbol{\Lambda}=\boldsymbol{U}^{M}$, where

$$
U_{i j}= \begin{cases}p & i=j \\ p^{j-i-1}(1-p)^{2} & i<j . \\ 0 & i>j\end{cases}
$$

Although $\boldsymbol{U}$ is not diagonalizable, we can still compute the $M$-th power using its 
Jordan decomposition. Using induction, it can be shown that $\boldsymbol{\Lambda}=\boldsymbol{T} \boldsymbol{J}^{\boldsymbol{M}} \boldsymbol{T}^{-\mathbf{1}}$, where

$$
\begin{aligned}
J_{i j}^{M} & =\left\{\begin{array}{ll}
p^{M-j+i}\left(\begin{array}{c}
M \\
j-i
\end{array}\right) i \leq j \\
0 & i>j
\end{array},\right. \\
T_{i j} & =\left\{\begin{array}{ll}
1 & j=i=1 \\
(-p)^{j-i}(1-p)^{-2(j-1)}\left(\begin{array}{c}
j-2 \\
j-i
\end{array}\right) & j \geq i \geq 2 \\
0 & \text { else }
\end{array},\right. \\
T_{i j}^{-1} & = \begin{cases}1 & j=i=1 \\
p^{j-i}(1-p)^{2(i-1)}\left(\begin{array}{c}
j-2 \\
j-i
\end{array}\right) & j \geq i \geq 2 . \\
0 & \text { else }\end{cases}
\end{aligned}
$$

After some manipulation, the final excitation probability becomes

$$
\begin{aligned}
P_{e f} & =\sum_{j=1}^{M} v_{j} P_{g i}^{(j)}, \\
v_{1} & =p^{M}=P_{\mathrm{LZ}}, \\
v_{j>1} & =p^{M+j-2} \sum_{t=0}^{j-2}\left(\begin{array}{c}
j-2 \\
t
\end{array}\right)\left[p q^{2(t+1)}\left(\begin{array}{c}
M \\
t+1
\end{array}\right)+\sum_{s=0}^{t} q^{t+s}\left(\begin{array}{c}
M \\
s
\end{array}\right)\right],
\end{aligned}
$$

where $q \equiv(1-p) / p$. Indeed, $v_{j}$ is the excitation probability when the environment is initially in the $j$-th state $\left(P_{g i}^{(j)}=1\right)$. Notice that for $P_{\mathrm{LZ}} \gtrsim e^{-M}, p \approx 1$ and hence $0<q \ll 1$.

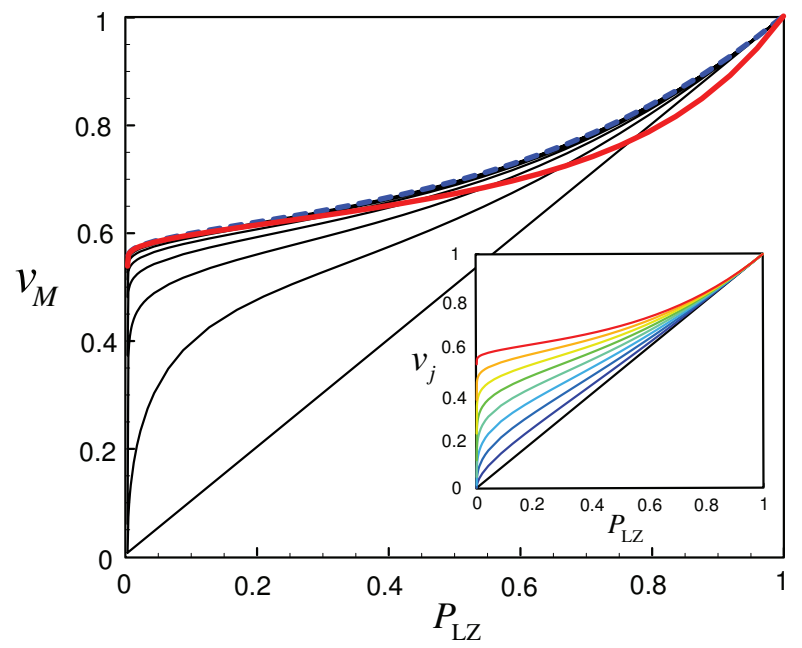

Fig. 2. $\quad v_{M}$ as a function of $P_{\mathrm{LZ}}\left(\equiv e^{-\pi g_{m}^{2} / 2 \nu}\right)$. The thin (black) lines are obtained using (17) with $m=0,1, \ldots, 7$, ordered from bottom to top. The thick dashed (blue) and solid (red) lines are exact and approximate $(M \rightarrow \infty)$ solutions using (21) and (22), respectively. Inset: $v_{j}$ vs $P_{\mathrm{LZ}}$ for $m=7$ and, from bottom to top, $j=1,16,32,48,64,80,96,112$, and 128 . 
The inset of Fig. 2 shows $v_{j}$ vs $P_{\mathrm{LZ}}$ for different values of $j$, when $m=7$. The initial rapid rise happens when $p\left(=P_{\mathrm{LZ}}^{1 / M}\right)$ changes from 0 to $\sim 1$, thus becomes extremely sharp at large $m$. Notice that $v_{j}$ monotonically increases as $j$ grows. This reflects the fact that higher energy levels have more paths available to them for excitation. Maximum excitation happens when the system starts in the highest energy level (i.e., $P_{g i}^{(M)}=1$ for which $P_{e f}=v_{M}$ ), hence $v_{M}$ gives an upper bound for the excitation probability $\left(P_{e f} \leq v_{M}\right)$. Figure 2 displays $v_{M}$ as a function of $P_{\mathrm{LZ}}$. As $m$ grows, $P_{\text {ef }}$ deviates from $P_{\mathrm{LZ}}$ ( $m=0$ case). The curves, however, saturate at $m \gtrsim 6$. This is important because it shows that there is always a nonzero ground state probability even when $m \rightarrow \infty$. All curves asymptotically join the $m=0$ curve as $P_{\mathrm{LZ}} \rightarrow 1$, indicating that in the fast passage limit the environment does not affect the excitation probability.

To find an analytical form for $v_{M}$ in the large $M$ limit, we use (17) and write $v_{M}=S_{0}+S_{1}$, where

$$
\begin{aligned}
& S_{0}=p^{2 M-1} \sum_{t=0}^{M-2} q^{2(t+1)}\left(\begin{array}{c}
M-2 \\
t
\end{array}\right)\left(\begin{array}{c}
M \\
t+1
\end{array}\right), \\
& S_{1}=p^{2 M-2} \sum_{t=0}^{M-2} q^{t}\left(\begin{array}{c}
M-2 \\
t
\end{array}\right) \sum_{s=0}^{t} q^{s}\left(\begin{array}{c}
M \\
s
\end{array}\right) .
\end{aligned}
$$

Using $\left(\begin{array}{c}M \\ s\end{array}\right)=\left(\begin{array}{c}M-2 \\ s\end{array}\right)+2\left(\begin{array}{c}M-2 \\ s-1\end{array}\right)+\left(\begin{array}{c}M-2 \\ s-2\end{array}\right)$, we have

$$
\begin{aligned}
S_{1} & =\frac{1}{2}\left[1+p^{2 M-2}\left(2-p^{-2}\right) W_{M}\right]-R_{M}, \\
W_{M} & =\sum_{t=0}^{M-2}\left(\begin{array}{c}
M-2 \\
t
\end{array}\right)^{2} q^{2 t}, \\
R_{M} & =p^{2 M-2} \sum_{t=0}^{M-2}\left(\begin{array}{c}
M-2 \\
t
\end{array}\right)\left(\begin{array}{c}
M-2 \\
t-1
\end{array}\right) q^{2 t+1} .
\end{aligned}
$$

Now, using

$$
\sum_{l=0}^{r}\left(\begin{array}{c}
m \\
l
\end{array}\right)\left(\begin{array}{c}
n \\
r-l
\end{array}\right) x^{2 l}=\frac{1}{2 \pi i} \oint_{C} \frac{(1+z)^{n}\left(1+x^{2} z\right)^{m}}{z^{r+1}} d z<x^{r-n}(1+x)^{m+n},
$$

which holds for $x>0$ and any closed contour $C$ around the origin, one can show that $S_{0}<p q \ll 1$ and $R_{M}<(p q)^{2} \ll 1$. Choosing an appropriate contour, (19) yields

$$
W_{M}=\frac{1}{2 \pi} \int_{-\pi}^{\pi}\left(1+2 q \cos \theta+q^{2}\right)^{M-2} d \theta
$$

As $M \rightarrow \infty,(1+2 q \cos \theta)^{M} \rightarrow P_{\mathrm{LZ}}^{-2 \cos \theta}$. Hence

$$
v_{\infty} \equiv \lim _{M \rightarrow \infty} v_{M}=\frac{1}{2}\left(1+\int_{-\pi}^{\pi} \frac{d \theta}{2 \pi} P_{\mathrm{LZ}}^{2(1-\cos \theta)}\right) .
$$

For $P_{\mathrm{LZ}} \ll 1$, we can further approximate the integrand as a gaussian to get

$$
v_{\infty} \approx 1 / 2+\operatorname{erf}\left(\pi \sqrt{-\ln \mathrm{P}_{\mathrm{LZ}}}\right) / 4 \sqrt{-\pi \ln \mathrm{P}_{\mathrm{LZ}}} .
$$


which gives $v_{\infty} \approx 1 / 2$ in the adiabatic limit $\left(P_{\mathrm{LZ}} \rightarrow 0\right)$. Equations (21) and (22) are plotted in Fig. 22 Equation (22) provides a good approximation for $v_{\infty}$ for a wide range of $P_{\mathrm{LZ}}$. This has a very important implication for AQC. It shows that there is always a nonzero probability of success bounded from below by $1-v_{\infty}$. In the adiabatic regime $\left(P_{\mathrm{LZ}} \ll 1\right)$, an environment of the sort modeled here can only suppress the probability of success by a factor close to 1/2.

\section{Numerical simulation}

We have also performed fully coherent simulations, using the Liouville equation 19

$$
\dot{\rho}(t)=-i[H(t), \rho(t)],
$$

for the density matrix $\rho$ of the system plus the environment. For a case with 4 environmental spins, plotted in Fig. 3, the coherent simulation shows only small fluctuations around the incoherent calculation, and also is very close to the equal gap calculation using (16). The figure also confirms that $v_{\infty}$ provides an upper bound for the excitation probability, as we expected. We have repeated this simulation for many other instances and obtained similar behavior.

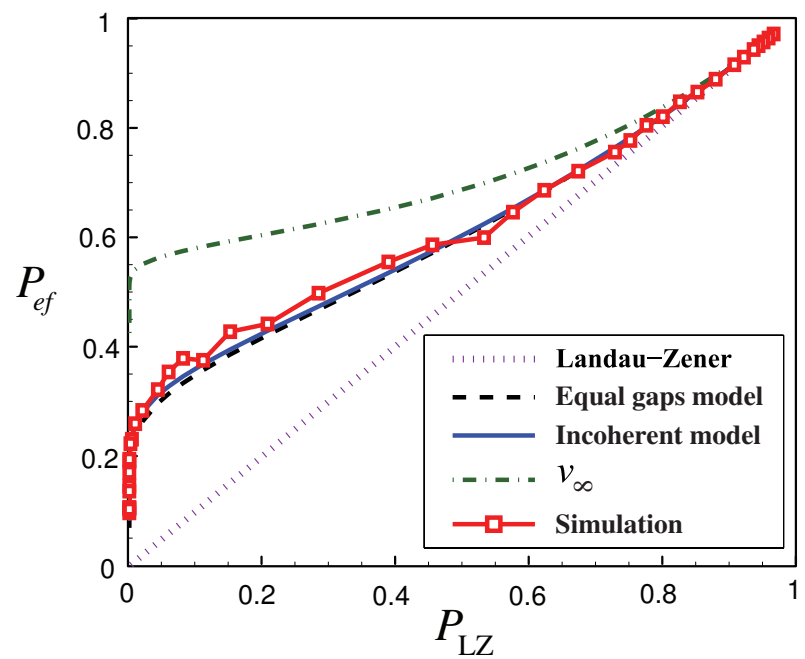

Fig. 3. Final excitation probability for completely coherent evolution and the approximate solutions. We assumed equally populated initial environmental states $\left(P_{g i}^{(j)}=1 / M\right)$. For the "incoherent", "equal gap", and " $v_{\infty}$ " curves we used (14), (16), and (17) respectively. Parameters are: $g_{m}=0.002,\left[B_{j}^{x}, B_{j}^{z}\right]=[0.08,-0.04],[-0.07,0.10],[0.15,-0.11],[-0.23,0.06], B_{j}^{y}=0$, and $J_{j}=0.148$ for all $m=4$ environmental spins.

\section{Conclusions}

We have shown that a $T=0$ noninteracting spin environment does not affect the transition probability in adiabatic quantum computation in the small gap regime. 
An excited (equilibrium or nonequilibrium) environment, on the other hand, will increase the excitation probability. We found that phase coherence does not play an important role in the excitation probability. Using an incoherent model with equal intermediate gaps, we identified a nonzero lower bound for the ground state probability. In the adiabatic limit, such a lower bound is $\sim 1 / 2$. AQC therefore is possible in the presence of such an environment.

\section{Acknowledgements}

The authors are grateful to A.J. Berkley, J.D. Biamonte, R. Harris, G. Martin, G. Rose, P.C.E. Stamp, C.J.S. Truncik, and M. Wubs for fruitful discussions.

\section{Appendix A. Adiabatic Grover search problem}

In this appendix, we systematically derive the effective two-state Hamiltonian for the case of adiabatic Grover search (AGS) algorithm. In AGS, as defined by Roland

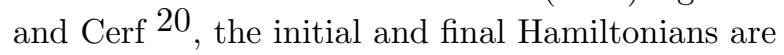

$$
\frac{H_{i}}{E}=\mathbb{I}-|+\rangle\left\langle+\left|, \quad \frac{H_{f}}{E}=\mathbb{I}-\right| \alpha\right\rangle\langle\alpha| .
$$

where $\mathbb{I}$ is the unity matrix, $|\alpha\rangle$ is the marked state, and

$$
|+\rangle=\frac{1}{\sqrt{N}} \sum_{l}|l\rangle
$$

with $|l\rangle$ representing states in the computation basis. Let us define a new state

$$
|\bar{\alpha}\rangle=\frac{1}{\sqrt{N-1}} \sum_{l \neq \alpha}|l\rangle .
$$

which is orthogonal to $|\alpha\rangle$. It is easy to see that $|+\rangle=(\sqrt{N-1}|\bar{\alpha}\rangle+|\alpha\rangle) / \sqrt{N}$. The Hamiltonians (A.1) can therefore be written completely in the subspace made of $|m\rangle$ and $|\bar{m}\rangle$ :

$$
H_{i} / E=\mathbb{I}-\frac{1}{N}\left(\begin{array}{cc}
1 & \sqrt{N-1} \\
\sqrt{N-1} & N-1
\end{array}\right), \quad H_{f} / E=\mathbb{I}-\left(\begin{array}{ll}
1 & 0 \\
0 & 0
\end{array}\right) .
$$

Using the Pauli matrices $\tau^{x, z}$ in the new 2-state subspace,

$$
H_{S}=(1-s) H_{i}+s H_{f}=-\frac{1}{2}\left(\tilde{\epsilon} \tau^{z}+\tilde{g}_{m} \tau^{x}\right),
$$

where

$$
\tilde{\epsilon}=\frac{E+(N-1) \epsilon}{N}, \quad \tilde{g}_{m}=\frac{\sqrt{N-1}}{N}(E-\epsilon),
$$

and $\epsilon=E(2 s-1)$. We have thrown away trivial terms proportional to $\mathbb{I}$. In the limit of large $N$, Hamiltonian (A.5) reduces to (3) with $g_{m}=E / \sqrt{N}$. 
Let us now introduce interaction between system qubits and environmental spins via the most general Hamiltonian

$$
H_{\mathrm{int}}=-\sum_{\alpha, \beta=x, y, z} \sum_{i=1}^{n} \sum_{j=1}^{m} J_{i j}^{\alpha \beta} \widetilde{\sigma}_{i}^{\alpha} \sigma_{j}^{\beta}
$$

Here $\widetilde{\sigma}_{i}$ and $\sigma_{j}$ denote Pauli matrices that act on the $i$-th qubit and $j$-th environmental spin, respectively, and $m$ is the number of the spins. In the subspace made of $|\alpha\rangle$ and $|\bar{\alpha}\rangle$, one can write the reduced interaction Hamiltonian by substituting.

$$
\begin{aligned}
& \tilde{\sigma}_{i}^{x} \rightarrow \frac{1}{N-1}\left(\begin{array}{cc}
0 & \sqrt{N-1} \\
\sqrt{N-1} & N-2
\end{array}\right), \\
& \tilde{\sigma}_{i}^{y} \rightarrow \frac{1}{N-1}\left(\begin{array}{cc}
0 & -i \sqrt{N-1} \\
i \sqrt{N-1} & N-2
\end{array}\right), \\
& \widetilde{\sigma}_{i}^{z} \rightarrow\left(\begin{array}{cc}
1 & 0 \\
0-1 /(N-1)
\end{array}\right) .
\end{aligned}
$$

Or in terms of Pauli matrices $\tau$

$$
\begin{aligned}
\tilde{\sigma}_{i}^{x, y} & \rightarrow \frac{N-2}{2(N-1)}\left(\mathbb{I}-\tau^{z}\right)+\frac{1}{\sqrt{N-1}} \tau^{x, y} \\
\tilde{\sigma}_{i}^{z} & \rightarrow \frac{N-2}{2(N-1)} \mathbb{I}+\frac{N}{2(N-1)} \tau^{z}
\end{aligned}
$$

In the large $N$ limit, one obtains

$$
\tilde{\sigma}_{i}^{x, y} \rightarrow-\frac{1}{2} \tau^{z}, \quad \tilde{\sigma}_{i}^{z} \rightarrow \frac{1}{2} \tau^{z},
$$

which yields

$$
H_{\text {int }}=-\frac{1}{2} \tau^{z} \sum_{\beta=x, y, z} \sum_{j=1}^{m} J_{j}^{\beta} \sigma_{j}^{\beta},
$$

where

$$
J_{j}^{\beta}=\sum_{i=1}^{n}\left(J_{i j}^{z \beta}-J_{i j}^{x \beta}-J_{i j}^{y \beta}\right) .
$$

Since the environmental spins are uncoupled, we can arbitrarily define the $z$-axis for each spin in such a way to obtain only longitudinal coupling:

$$
H_{\mathrm{int}}=-\frac{1}{2} \tau^{z} \sum_{j=1}^{m} J_{j} \sigma_{j}^{z},
$$

where

$$
J_{j}=\sqrt{\left(J_{j}^{x}\right)^{2}+\left(J_{j}^{y}\right)^{2}+\left(J_{j}^{z}\right)^{2}} .
$$

This result is valid more generally than for the spin environment studied here. In other words, at least for the adiabatic Grover search problem, any environment that 
couples to the individual qubits in a most general way would result in a longitudinal coupling of the effective two-state system (made of the lowest two energy states) to the environment. This can be clearly seen from Eqs. (A.8) and (A.9) that are independent of the type of environment. Notice that in (A.8) the terms involving $\tau^{x, y}$ always appear with a coefficient of $O(1 / \sqrt{N})$ and therefore will vanish in the limit $N \rightarrow \infty$. It physically makes sense because such off-diagonal terms in the interaction Hamiltonian would cause relaxation between the two levels with a rate of $O(1 / N)$. This means solving the search problem just based on classical relaxation to the ground state (i.e., classical annealing) will take a time of $O(N)$ which is the complexity of the classical search. One therefore would expect that the same property also persist for problems that are more general than the adiabatic Grover search; otherwise, e.g., spin glass problems could be solved efficiently by just letting the system relax to the ground state.

\section{References}

1. A. J. Leggett et al., Rev. Mod. Phys. 59 (1987) 1.

2. N. Yamada, H. T. A. Sakuma, J. App. Phys. 101 (2007) 09C110.

3. N. V. Prokof'ev and P. C. E. Stamp, Rep. Prog. Phys. 63 (2000) 669.

4. G. Falci et al., Phys. Rev. Lett. 94 (2005) 167002.

5. A. Shnirman et al., Phys. Rev. Lett 94 (2005) 127002.

6. A. Morello, P. C. E. Stamp, and I. S. Tupitsyn, Phys. Rev. Lett. 97 (2006) 207206.

7. A. M. Childs, E. Farhi, and J. Preskill, Phys. Rev. A 65 (2001) 012322.

8. J. Roland and N. J. Cerf Phys. Rev. A 71 (2005) 032330.

9. E. Farhi, J. Goldstone, S. Gutmann, J. Lapan, A. Lundgren, and D. Preda, Science 292 (2001) 472.

10. L. D. Landau and E. M. Lifshitz, Quantum mechanics, non-relativistic theory, (Pergamon Press, 1977).

11. R. W. Simmonds et al., Phys. Rev. Lett. 93 (2004) 077003.

12. J. M. Martinis et al., Phys. Rev. Lett. 95 (2005) 210503.

13. L. Faoro et al., Phys. Rev. Lett. 95 (2005) 046805.

14. R. H. Koch, D. P. DiVincenzo, and J. Clarke, cond-mat/0702025

15. P. Ao and J. Rammer, Phys. Rev. B 43 (1991) 5397.

16. Y. Kayanuma and H. Nakayama, Phys. Rev. B 57 (1998) 13099.

17. M. Wubs et al., Phys. Rev. Lett. 97 (2006) 200404.

18. N. A. Sinitsyn and N. Prokof'ev, Phys. Rev. B 67 (2003) 134403.

19. K. Blum, Density Matrix Theory and Applications (Plenum Pub. Corp. 1981).

20. J. Roland and N. J. Cerf, Phys. Rev. A 65 (2002) 042308. 